\title{
VARIOUS MANIFESTATIONS OF NON-SPECIFIC ARACHNOIDITIS OF INDEFINITE AETIOLOGY
}

\author{
By N. G. HULBERT, M.A., M.D., M.R.C.P. \\ (Temporary Assistant Physician, West End Hospital for Nervous Diseases; - \\ Temporary Physician, Metropolitan Hospital, etc.)
}

\section{Introduction}

This article is not intended as a complete review of this subject, nor as a detailed report or analysis of all the examples of the condition treated at the West End Hospital for Nervous Diseases. It seemed, however, that a short note on the subject might be of interest, as many of the conditions included in this group are amenable to treatment by modern neurosurgery, though they may be somewhat rare. It further appeared that it would be of interest to Members of the Fellowship of Medicine to illustrate it by brief reference to some of the cases which have been discussed with them from time to time at the M.R.C.P. courses at the West End Hospital, without, however, prejudicing any full report or analysis of such cases, which may be published elsewhere at a later date.

\section{Definition}

The term arachnoiditis has come to be applied to an end process without an associated active disease, and does not imply any active inflammation. The condition consists of adhesions or cysts in the subarachnoid space between the arachnoid and the pia mater.

Similar adhesions may be formed overlying a tumour, or in association with an active syphilitic meningitis, but on the basis of our definition such cases would not be regarded as falling in the category of arachnoiditis.

\section{Aetiology}

Such adhesions may arise as the result of any exudate in the subarachnoid space from subsequent organisation and fibrosis, occasionally with the formation of loculated spaces or cysts. There is an example of such a spinal cyst, which has often been shown to Members of the Fellowship of Medicine, in the Museum of the West End Hospital, and an excellent photograph of the specimen appears on p. I022 of the I943 edition of Beattie and Dickson's Textbook of Pathology. If such cysts are formed, and if they contain fluid with a higher protein content than that of the cerebrospinal fluid, they may subsequently swell by absorbing fluid by osmosis and so produce local or general pressure signs, and clinically resemble spinal cord tumours.

The following are some of the possible causes of such exudates:

(I) Trauma either at birth or in later life may give rise to a localised bruising of the nervous system with exudation of blood and serum into the subarachnoid space within the cranium. This may be either in the region of the trauma or the result of a contre-coup lesion, hence trauma to the vertex of the skull may produce an exudate round the optic chiasma at the base of the brain.

Again, the introduction of the irritant substances into the subarachnoid space may produce adhesions; for example, the accidental introduction of the soluble highly alkaline sulphonamide products before their nature was fully appreciated may produce subsequent adhesions as well as immediate toxic damage. Some have also incriminated lipiodol and its various substitutes, particularly samples which have been kept overlong in stock and contain free iodine, though opinion is divided on this point.

(2) Inflammatory exudates may also fail to absorb completely. Thus in pyogenic and in particular meningococcal menigitis residual adhesions may be left; again in areas adjacent to a localised periositis or diseased bone adhesions may form in the subarachnoid space, for example, in the vicinity of an infected nasal sinus.

(3) Spontaneous haemorrhages in the subarachnoid space may also fail to absorb completely. These may occur in such haemorrhagic diseases as purpura or scurvy, or following a leak from an intracranial aneurysm or atheromatous vessel. 
Symptoms of arachnoiditis may arise in the following ways:-

(i) A band of fibrous tissue or an arachnoidal cyst may exert local pressure on the nervous system, for example on the optic chiasm or on the spinal cord.

(ii) An arachnoidal cyst may act as a space occupying tumour producing local symptoms, but only rarely will it raise the intracranial pressure sufficiently to produce generalised pressure symptoms of papilloedema, headache and vomiting.

(iii) An arachnoiditis may obstruct the flow of cerebrospinal fluid and in the skull cause a raised pressure and hydrocephalus, which may mimic a cerebral tumour, and in particular cause a secondary pituitary dysfunction, or in the vertebral canal interfere with the nutrition of the cord.

(iv) The adhesions may press on the surface vessels, especially on the veins of the nervous system and so lead to their thrombosis with consequent damage to the nervous tissue drained by them.

(v) There may be traction on the nervous system either in general by the contracture of $\bar{\omega}$ the scar tissue, or in the skull as a result of movements of the head, when the movements of $\frac{D}{0}$ the brain in the C.S.F. may not be quite synchronous with those of the surrounding arachnoid and dưra mater.

\section{SOME OF THE MAIN CLINICAL GROUPS OF ARACHNOIDITIS}

(I) Subarachnoid adhesions, which are usually vascular, may be formed over the cerebral cortex. Some cases of post-traumatic epilepsy fall into this group, other than those which result from local cortical scaring or post-traumatic cortical atrophy.

Thus a female patient, aged 22, was first in seen June 1942 at the Metropolitan Hospital. Six years previously she had had a motor bicycle accident, after which she said that she had remained unconscious for 3 weeks. She sustained a fractured skull and had a transitory left-sided hemiplegia. She remained 3 months in hospital; 8 months later she started to have left-sided Jacksonian epileptic attacks, which started in the region of the mouth and proceeded to generalised fits with loss of consciousness. She had had a period of 2 years fairly free from fits, but more recently they had been recurring. On examinatio the central nervous system showed no abnormality, and she was admitted to the West End Hospitar. for further investigation in October 1942. Skull X-ray showed a probable united fissure fracture in the right temporal region. The electro-encephalogram was within normal limits, thus tending to exclude an underlying idiopathic epilepsy or a generalised cortical atrophy. The air encephalography showed the temporal horn of the $R$. Jateral ventricle to be drawn down and back, and the posterior horns to be drawn to the right.

Mr. G. C. Knight operated, making a low right temporal flap, and found some atrophy of the convolutions of the temporal lobe, to which were attached some 15 vascular adbesions, thus accounting for the distortion of the air-encephalogram. He divided these, and inserted cellophane to prevent their reforming. When seen a year later she had remained free from fits.

Operation in such cases would seem justified if the fits are süfficiently frequent and disabling, if the electro-encephalogram shows either a focal lesion or a normal tracing, and if the air-encephalogram shows a deviation of the ventricular system suggesting traction by adhesions.

(2) A condition which used to be known as meningitis serosa circumscripta, namely, adhesions or cysts pressing on the spinal cord, may produce symptoms of tumour, and also further damage it by causing venous thromboses.

As examples of this condition I would cite two cases.

A man, aged 58, was first seen in 1940 at the Metropolitan Hospital with a progressive history of 2 years numbness of the right leg and 6 months of pains and a feeling of heaviness in the right leg. On exami- $\frac{7}{0}$ nation pain and temperature sense were lost in the right leg up to the level of the first lumbar segment, $三$ with a little impairment of sensation to about the level of the 8th dorsal segment. The tendon reflexes on the $\mathrm{N}$ left leg were exaggerated and greater than those of the right side, and the left plantar response was extensor. Deep pressure and vibration sense, however was lost in the right and not in the left leg, so $N$ the picture was not quite that of a Brown-Séquard syndrome. On admission to the West End Hospital N the Queckenstedt test showed a delayed rise and fall of the C.S.F. pressure, and lipiodol injected by $\omega$ cisternal puncture showed on the X-ray a partial block at the level of the 6th dorsal vertebra. Mr. G. C. 0 Knight operated in February 1940 and found a broad sheet of massive adbesions, which he divided, 0 opposite the lower border of the 6 th dorsal vertebra. The cord itself appeared quite normal, and there $\frac{C}{\Phi}$ was no underlying meningioma, neurofibroma, or intervertebral disc to cause adhesions. The man's $\stackrel{\oplus}{\rightarrow}$ condition remains satisfactory at the present time, and the progress of the disorder has undoubtedly : been arrested, though the physical signs remain the same, for it is now nearly 4 years since the operation. The origin of the adhesions in this case remains in doubt, but it seems possible that they may have been the sequel of some forgotten trauma. 
The second case is of a woman, aged $2 \mathrm{r}$, who was admitted by Dr. Worster-Drought to the West End Hospital on June I, 1940. Her history was that in February I940 she contracted meningococcal meningitis, which for the first 3 weeks was thought to be influenza. After admission to the West End Hospital she developed a complete paraplegia in flexion with sensory loss to the level of the 5 th or 6 th dorsal segments.

Lumbar puncture on 3 occasions gave a true dry tap, no fluid being obtained. On June IIth Mr. G. C. Knight did a laminectomy, removing the arches of the $2 \mathrm{nd}$, 3rd, 4th, and 5 th dorsal vertebrae, and found a band of adhesions, which he removed, opposite the 4 th dorsal vertebra. When, however, a rubber catheter was passed down the subarachnoid space it met with an obstruction at a lower level suggesting further adhesions. On June I4th she had improved and was able to move all her toes, and had $30^{\circ}$ of flexion and extension at the hips with slight power of flexion of the knees. Then on July 3 rd Mr. G. C. Knight did a further laminectomy, removing the arches of the 6th, 7th, 8th, 9th, Ioth, and I I th vertebrae. Dense plastic adhesions were found, causing complete obliteration of the subarachnoid space. There were also appearances suggestive of some thrombosis of the vessels of the cord. After the adhesions were divided and the cord freed a catheter could be passed $2 \frac{1}{2}$ inches down from the conus. After the operation the sensory level slowly descended till the sensation was practically normal by September I940, when she recovered control of the bladder. On her discharge from hospital in March I94I she was just able to walk without a stick, and was walking fairly well with a stick. She has since further improved in her walking, being able to lead a fairly normal life, though on examination there are still signs of pyramidal tract damage in the legs. This remarkable degree of recovery fully justified the extensive operations.

(3) Arachnoiditis in the region of the optic chiasm may produce pressure on it or on the optic nerves, so causing progressive failure of vision with the appearance of optic atrophy on ophthalmoscopic examination. The diagnosis is largely made by exclusion.

Three such cases with a review of the subject have been published by Goldsmith in the section of ophthalmology of the Proceedings of the Royal Society of Medicine in February I943; he includes some good illustrations of the condition taken from the French literature. Goldsmith analysed the French series of I29 cases and found that a central scotoma occurred in $3 I$ per cent, a concentric contraction of the visual fields in 23 per cent, and temporal loss of the fields in I7 per cent, while others showed nasal or horizontal loss or homonymous hemianopia: The ophthalmoscopic appearances in order of frequency were primary optic atrophy, postpapillitic atrophy, papilloedema and temporal pallor, while in Io per cent of cases the fundi were normal. The progress of the condition tended to be by successive exacerbations. After operation, which gives a variable degree of recovery, there may be deterioration, but this is rare later than a month after operation.

Mr. G. C. Knight has recently operated on three cases of this condition at the West End Hospital. One of them, a woman under the care of Dr. Worster-Drought, has been shown on more than one occasion to the Members of the Fellowship of Medicine, and the diagnosis discussed, before operation was finally decided upon.

This woman, aged 34, was admitted in August I94I, with a history that one year before she had suddenly found that she was unable to read, the print being blurred when she looked at it. This failure of vision got progressively worse until on admission her visual acuity was R.6/36 L.6/18. The visual fields showed a generalised peripheral contraction; the optic discs showed early bilateral primary optic atrophy, while the C.S.F. and the X-rays of the skull and optic foramina were all normal. She was re-admitted in October I94I, when the visual acuity was $R .6 / 24$ and L.6/I8. The discs were unchanged. She had some dental extractions with a view to removing sepsis, and an air-encephalography showed normal X-ray appearances of the ventricular system. Dr. Worster-Drought suggested the diagnosis of chiasmal arachnoiditis, but a major operation on the head seemed rather a severe measure at this stage.

On re-admission in March 1942 the visual fields were slightly more constricted, the optic discs were whiter and the visual acuity R.6/36, L.6/60. In September I942 she said that she was worse and unable to see the names or the numbers on the front of trams; the visual acuity was R.6/6o, L.6/6o, and she had lost her perception of colour. The visual fields were grossly constricted on September 5 th, only a very small area $10^{\circ}$ to $15^{\circ}$ from the macula in the centre of the field being retained.

Mr. G. C. Knight operated on October I 3 th, using a right frontal bone flap. He found light adhesions round the right optic nerve and over the dorsal aspect of chiasm, and thickening of the arachnoid of the cisterna chiasmalis, so that it formed a sleeve around the optic nerves. The adhesions were broken down, and the optic nerves and the chiasm freed. After the operation the appearance of the discs was unchanged, but the visual fields showed a remarkable recovery, becoming almost full by the I Ith of November. It seems, therefore, that this diagnosis should always be kept in mind in considering cases of progressive failure of vision and optic atrophy.

\section{CONCLUSION}

It used to be said that neurology had little or no treatment to offer, and was a matter of diagnosis and prognosis. As a result of present-day advances however, I think that it may 
be said to compare quite favourably in this respect with other branches of medicine, such as cardiology. I hope, therefore, that this subject may be of interest, particularly in view of the therapeutic possibilities, of modern neurosurgery.

I wish to thank the Staff of the West End Hospital for Nervous Diseases for permission to refer to case notes, and in particular Dr. Worster-Drought, and Mr. G. C. Knight. I would also emphasise that the diagnosis and treatment of such cases is essentially a marter of team work on the part of the Hospital Staff.

\title{
CYSTICERCOSIS
}

\author{
By R. E. VERNEY, M.B., F.R.C.P.E., D.R. \\ (Assistant Physician, Royal Infirmary, Edinburgh)
}

Although Leuckart (I856) first elucidated the life cycle of tania solium, it was not until I933 that the pioneer work of W. P. MacArthur drew attention to the importance and prevalence of cysticercosis as a clinical entity in this country: with the collaboration of $\mathrm{H}$. B. F. Dixon, D. W. Smithers and W. K. Morrison working at the Queen Alexandra Military Hospital, Millbank, he worked out the clinical features, pathological changes, and radiographic appearances of cysticercosis. He drew attention to the prevalence of the condition amongst soldiers who had been stationed abroad-particularly in India. As tænia solium infection of pigs and man is common on the continent of Europe, the Middle East, India, and the Far East, it is certain that a large number of our fighting personnel will return after the war with the unpleasant manifestations of this infection. The condition of cysticercosis must therefore always be kept in mind when dealing with a patient who has resided abroad, and who has developed such manifestations as fits, symptoms suggesting some degenerative process of the nervous system or a psychic disorder.

A picture of the pathological processes and the protean manifestations of the condition of cysticercosis may be visualised by the consideration of the following typical case.

W.C., æt 44, commenced his adult life in the Regular Army, serving on the North-West Frontier o India from 1918 to 1924 . During that period he had several attacks of what was considered to be malariat - bouts of fever, with intense headaches and severe pains in his muscles. After returning to this country he suffered from repeated epileptiform fits from 1927 to 1933 . Early in 1942 he was referred to hospital with a diagnosis of left renal colic, as he had suffered for a period of four months from sudden attacks of violent and commanding pain which commenced in the left lumbar region and radiated round the abdomen to the area of the umbilicus. The duration of each attack varied from a few minutes to several hours. With the increasing frequency and severity of the attacks he had noticed that pain of a similar character and distribution was being radiated to the right side to encircle the trunk, suggestive rather of the girdle pains of tabes. After each attack he would feel exhausted and pass into a deep sleep for several hours. He denied any possibility of a syphilitic infection in early adult life, the reflexes in his lower limbs were found to be intact, his pupillary reflexes were normal, and there was no disorder of sensation. The urine was free of red cells, but in order to exclude the possibility of a renal calculus an $\mathrm{X}$-ray examination of the renal tract was carried out. The film showed, dotted about the abdominal $\exists$ area, numerous small well-defined elongated opacities having the characteristic features of calcified $\dot{0}$ cysticerci. Further films of the entire body demonstrated the presence of numerous calcified cysticerci scattered through the musculature of the extremities; one was demonstrated in the skin, but there were none visible in the brain or spinal cord.

Incidence in Great Britain.

As the result of a wise provision of Parliament in the year 1582 , which made the sale of $\frac{D}{O}$ "mesell pork" a crime in this country, the tænia solium virtually does not exist in England and Wales, or in Scotland. In parts of Ireland where the social circumstances of the people $\tilde{O}$ are more primitive it is not uncommon. In Dixon and Smithers' review of seventy-one cases of ' cysticercosis (which included those reported in the literature) only one appeared to have been 0 acquired in this country. Tænia saginata, which is seen so commonly, produces eggs which $\omega$ are morphologically identical with those of the pig worm, but does not utilise man as the intermediate host for the cysticercus stage.

\section{Mode of infection.}

This patient became infected in one of two ways. He may have been the host of a taenia solium as the result of eating measly pork which had been insufficiently cooked. He could then 